
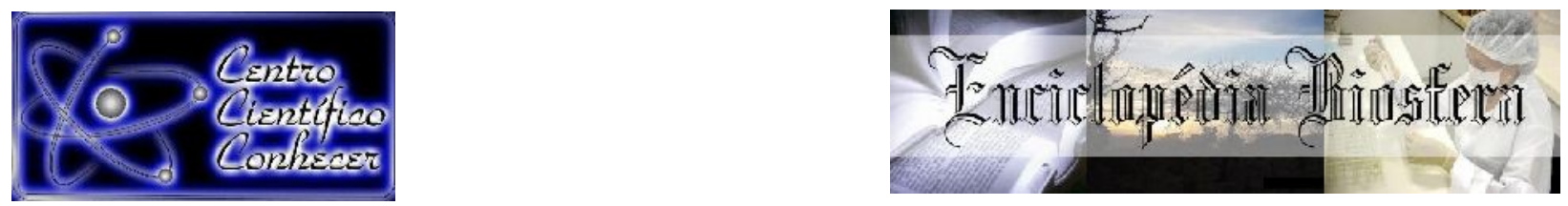

\title{
Eichhornia azurea COMO HOTSPOT PARA MACROINVERTEBRADOS AQUÁTICOS: FERRAMENTA PARA A APLICAÇÃO DE ÍNDICES DE AVALIAÇÃO AMBIENTAL
}

\author{
Acisa Raimunda de Souza1; Claumir Cesar Muniz ${ }^{1}$; Ernandes Sobreira Oliveira \\ Junior $^{1}$
}

${ }^{1}$ Mestranda em Ciências Ambientais pela Universidade do Estado de Mato Grosso.

UNEMAT- Centro de Limnologia Biodiversidade e - Etnobiologia do Pantanal - CELBE - Campus Universitário de Cáceres, 78200-000- Cáceres, MT

2 Professor Doutor em Ecologia e Recursos Naturais. Universidade do Estado de Mato Grosso Laboratório de Ictiologia do Pantanal Norte - LIPAN. Campus Universitário de Cáceres, 78200-000Cáceres, MT

${ }^{3}$ Professor Doutor em Ciências pela Universidade de Radboud - Holanda. UNEMATUniversidade do Estado de Mato Grosso - Laboratório de Ictiologia do Pantanal Norte - LIPAN. Campus Universitário de Cáceres, 78200-000- Cáceres, MT

Autor para correspondência: cisa.rsouza@gmail.com

Recebido em: 22/09/2018 - Aprovado em: 23/11/2018 - Publicado em: 03/12/2018 DOI: 10.18677/EnciBio_2018B85

RESUMO
Geologicamente novo, o Pantanal apresenta uma biodiversidade que mescla Amazônia e Cerrado. Poucos estudos sobre a macrofauna fitófila dessa região foram executados, deixando uma lacuna de conhecimento a respeito da composição fitófila. Ainda, a grande abundância de macrófitas aquáticas como Eichhornia azurea, as quais formam grandes bancos e estão presentes em sistemas lênticos pantaneiros, servem também como micro-habitat para o estabelecimento da comunidade da macrofauna, a qual pode sofrer uma mudança devido ao ciclo hidrológico. Desta forma, este estudo teve o objetivo de identificar a macrofauna fitófila associada à $E$. azurea, de diferentes baías do Pantanal durante quatro períodos hidrológicos. Foram realizadas coletas mensais de caule e raízes da macrófita, bem como dados limnológicos de oxigênio dissolvido, $\mathrm{pH}$, temperatura e transparência. Os macroinvertebrados associados à macrofita aquática foram identificados ao nível taxonômico de família, e avaliados para obtenção do índice de conservação das baías, através do índice de diversidade (Shannon- Wiener), homogeneidade e o índice biótico Biological Monitoring Working Party (BMWP) para biomonitoramento. Foram quantificados 4,164 espécimes pertencentes a 42 famílias em 12 grandes grupos. Chironomidae (Díptera) foi a família mais abundante em todos os locais de coletas e períodos sazonais. O índice de diversidade de ShannonWienner variou entre 0.94 e 1.08, e o BMWP indicou a qualidade da água como ótima em todas as baías estudadas. A diversidade da macrofauna associada muda conforme a estação do ano, indicando que a estrutura da comunidade depende dos fatores ambientais externos. Assim, a utilização do índice de biomonitoramento para a análise da qualidade das baías do Pantanal demonstra que organismos associados à macrófitas aquáticas também podem ser utilizados como uma ferramenta robusta para a geração de resultados que integrem a identificação ambiental.

Palavras- Chave: Índice De Biomonitoramento. Macrofauna. Pantanal. ENCICLOPÉDIA BIOSFERA, Centro Científico Conhecer - Goiânia, v.15 n.28; p. 1043 


\title{
Eichhornia azurea AS HOTSPOT FOR AQUATIC MACROINVERTEBRATES: TOOL FOR THE ENVIRONMENTAL EVALUATION INDEX APPLICATION
}

\begin{abstract}
Geologically recent, Pantanal presents the biodiversity merging Amazon and Cerrado. Few studies on macrofauna were done in this region, leaving a great hole about the phytophile composition. Furtherlmore, the great abundance of macrophytes, such as Eichhornia azurea which forms huge beds and is present in the lentic systems of the Pantanal, has the role of also host organisms of the macrofauna community, which can suffer with any hidrological cycle changes. Therefore, we conducted a study aiming to identify the phytophile macrofauna associated to $E$. azurea, from different bays and hydrologic periods in Pantanal. Samples were taken monthly containing stem and roots of the macrophytetogether with limnological data of dissolved oxygen, $\mathrm{pH}$, temperature and transparency. The macroinvertebrates associated to the macrophyte were screened to the family taxonomic level in order to obtain the diversity index (Shannon-Wiener), homogeneity and Biological Monitoring Working Party (BMWP) for biomonitoring. In total, 4,164 specimens belonging to 42 families in 12 large groups were identified. Chironomidae (Diptera) was the most abundant family in all of the sample sites and seasons. The Shannon-Wienner index varied from 0.94 to 1.08 and the BMWP indicated that the systems presented good water quality in all of the studied bays. The macrofauna diversity changes according to the season, indicating that the community structure also depends on the environmental parameters. Therefore, the uses of biomonitoring indexes in order to obtain the environmental quality of the bays in the Pantanal denotes that the organisms hosted by macrophytes can also be a tool to generate results for the environmental analysis.
\end{abstract}

Keywords: Biomonitoring Index. Macrofauna. Wetland.

\section{INTRODUÇÃO}

O Pantanal é formado por diferentes corpos d'água (FROTA et al., 2017) como as baías que são diretamente afetadas pelo pulso de inundação, e impacta a planície de inundação do Pantanal, não sendo ainda totalmente compreendido como as enchentes e secas afetam biota (THOMAZ et al., 2003).

As macrófitas aquáticas desenvolvem papel importante na manutenção dos sistemas de inundação (PAGIORO; THOMAZ, 1999), e um efeito em cascata ocorre em virtude de alterações que afetem sua comunidade no que tange os demais organismos associados como peixes, macroinvertebrados e zooplâncton (THOMAZ et al., 2003).

A macrófia aquática Eichhornia azurea é comum em áreas litorâneas (BAZZANTI et al., 2010), e favorece a presença abundante e rica da macrofauna, sendo utilizada como fonte alimentar para espécies de macroinvertebrados fragmentadores e coletores (MARTINS et al., 2011). A presença desses macroinvertebrados no ambiente também denota a sua qualidade e 0 seu funcionamento (BARBA-ÁLVAREZ et al., 2013), em que dependendo do serviço ecossistêmico que o grupo desempenha, sua qualidade pode ser determinada (Romero et al 2017). Além disso, devido a baixa mobilidade e a alta susceptibilidade às alterações ambientais locais (CORTEZZI et al., 2009), esses organismos são amplamente utilizados na caracterização ambiental através dos índices de qualidade ambiental (ROLDÁN-PEREZ, 2016; ROMERO et al., 2017). Dentre os índices de qualidade ambiental destaca-se o Biological Monitoring Working Party (BMWP), ENCICLOPÉDIA BIOSFERA, Centro Científico Conhecer - Goiânia, v.15 n.28; p. 1044 
frequentemente utilizado para avaliação da qualidade da água utilizando macroinvertebrados, e se apresenta como ferramentas fácil e útil para o monitoramento da qualidade do sistema (Barbola et al. 2011; Yoshida e Uieda, 2013).

Assim, esses organismos se tornam uma peça importante na avaliação da qualidade hídrica, principalmente levando em consideração as alterações ambientais decorrentes no Pantanal como o desmatamento (RODRIGUES-JUNIOR et al., 2013; PERES et al., 2016), queimadas (PAIVA et al., 2014), construção de pequenas centrais hidrelétricas nos rios adjacentes ao rio Paraguai (SOUZA-FILHO, 2013), as quais contaminam as águas (PIRES et al., 2014) e consequentemente leva a perda da biodiversidade.

Mudanças no entorno do Alto Pantanal podem estar afetando as baías marginais ao rio Paraguai, corroborando assim para a perda, ou mudança na biodiversidade de macroinvertebrados aquáticos associados a macrófitas aquáticas. Desta forma, este trabalho objetiva identificar os macroinvertebrados associados à Eichhornia azurea em baías marginais ao rio Paraguai e avaliar a qualidade ambiental de acordo com índices de estrutura de comunidades e de biomonitoramento, fornecendo assim dados que identifiquem o ambiente pantaneiro.

\section{MATERIAL E MÉTODOS}

Coletas mensais em seis lagoas marginais ao rio Paraguai, um dos principais rios do bioma Pantanal, que receberam nominação por numerais de um a seis, estão situadas entre o perímetro urbano de Cáceres e a Estação Ecológica de Taiamã.

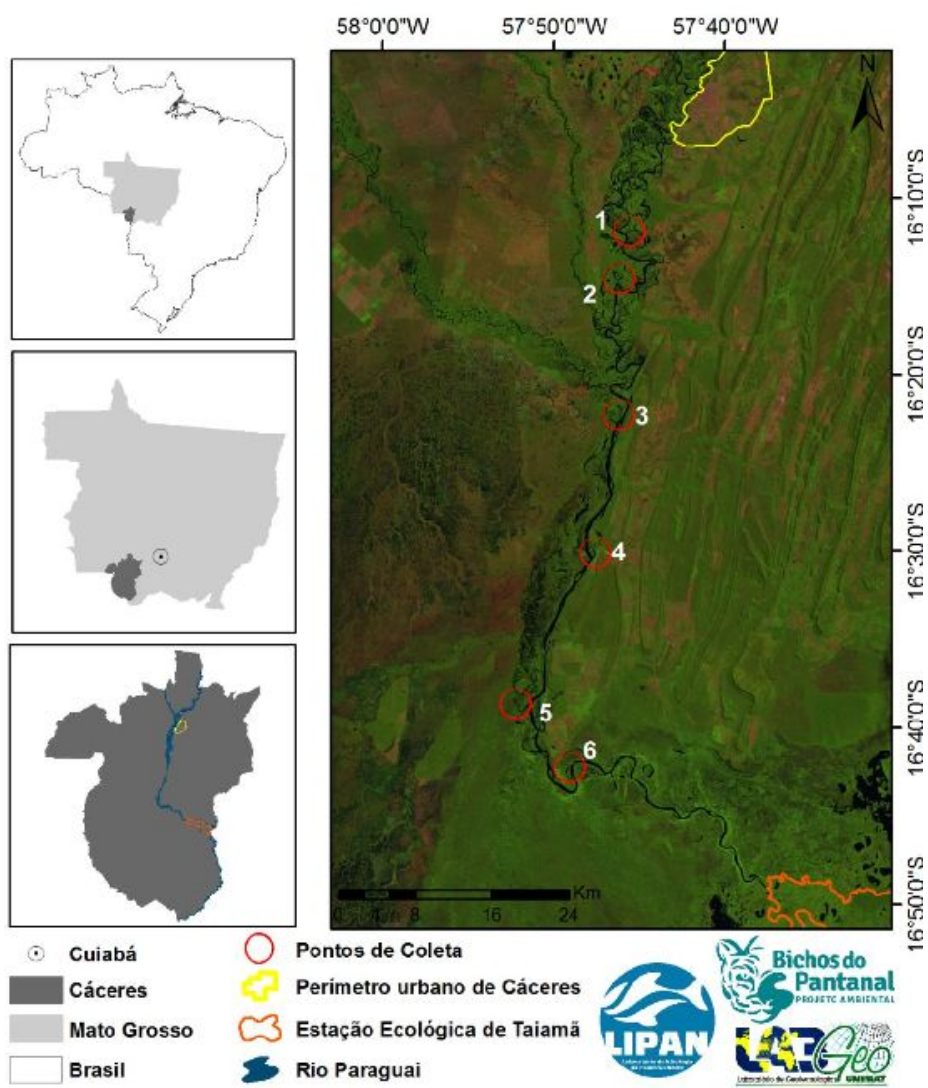

Figura 1. Identificação das seis baías situadas no Rio Paraguai, no município de Cáceres, Mato Grosso, Brasil.

Fonte: Laboratório de Geotecnologias da Universidade do Estado de Mato Grosso- UNEMATCáceres (2014) 
As coletas foram realizadas durante 10 meses, compreendendo os diferentes períodos sazonais, cheia (março), vazante (abril, maio, junho), estiagem (julho, agosto, setembro) e enchente (outubro, novembro, dezembro). Para a coleta do material biológico foram selecionados bancos de Eichhornia azurea $\left( \pm 30 \mathrm{~m}^{2}\right)$ presentes nas margens das baías laterais do rio Paraguai. As baías laterais foram selecionadas considerando a distância com a cidade de Cáceres.. Os órgãos da planta, caule e raízes, foram cortadas com utilização de uma tesoura de poda (de acordo com metodologia aplicada por SAULINO; TRIVINHO-STRIXINO, 2014) a partir do terceiro entre nó, garantindo a estabilidade do nicho amostral e a submersão dos órgãos coletados. Após coletadas, as amostras foram acondicionadas em recipientes plásticos com água do meio e contendo $10 \%$ de formol para a conservação. Foram coletados ainda os seguintes parâmetros limnológicos: pH, oxigênio dissolvido (OD), temperatura da água, transparência da coluna d'água.

As amostras foram encaminhadas para o laboratório de Ictiologia do Pantanal Norte - LIPAN, da Universidade do Estado de Mato Grosso - UNEMAT, no Centro de Limnologia, Biodiversidade e Etnobiologia do Pantanal - CELBE. Os Macroinvertebrados foram identificados até o nível taxonômico de família, com o auxílio de manuais de identificação segundo CALOR, 2007; MUGNAI et al., 2010; e PÉREZ, 1988. Para a obtenção do índice de qualidade ambiental (BMWP; Tabela 1), e dos índices de diversidade (Shannon-Wiener, $\mathrm{H}^{\prime}$ ), e de homogeneidade $(\mathrm{IH})$, os organismos foram quantificados por local (baía) e período sazonal (meses) de coleta sob lupa estereoscópica.. Os índices de Shannon-Wiener e Homogeneidade foram calculados com o programa Bioestat versão 5.0. O índice BMWP para cada baía calculado segundo a equação:

$$
B M W P=\Sigma n i+n+\sum n j
$$

Onde:

ni: é o número de indivíduos da família

$\mathrm{n}$ : é a nota que representa a família no BMWP

$\sum n j$ : é a somatória das notas recebidas pelas outras famílias presentes na baía.

TABELA 1 - Classes de qualidade, significado dos valores do bmwp e cores (adaptado de ALBA-TERCEDOR; ORTEGA, 1988).

\begin{tabular}{|c|c|c|c|c|}
\hline Classe & Qualidade & Valor & Significado & Cor \\
\hline I & Boa & 100 & $\begin{array}{l}\text { Águas muito limpas, ou não } \\
\text { alteradas de modo sensível }\end{array}$ & Azul \\
\hline II & Aceitável & $61-100$ & $\begin{array}{l}\text { São evidentes alguns efeitos } \\
\text { de Contaminação. }\end{array}$ & Verde \\
\hline III & Duvidosa & $36-60$ & Águas contaminadas. & Amarelo \\
\hline IV & Crítica & $16-35$ & Águas muito contaminadas. & Laranja \\
\hline V & Muito crítica & $\leq 15$ & $\begin{array}{ll}\text { Águas } & \text { fortemente } \\
\text { contaminadas. } & \end{array}$ & Vermelho \\
\hline
\end{tabular}




\section{RESULTADOS E DISCUSSÃO}

Os dados demonstram a existência de 42 famílias pertencentes a 12 grupos foram registradas, como segue: Achari, Bivalva, Coelencerata, Coleoptera, Diptera, Ephemeroptera, Gastropoda, Hemiptera, Hirundinea, Odonata, Oligochaeta e Trichoptera, num total de 4.164 indivíduos. As famílias mais abundantes são representadas na tabela 2 .

TABELA 2- Média (DP) de ocorrência das famílias de acordo com os diferentes períodos sazonais do ciclo hidrológico pantaneiro no ano de 2014 ..

\begin{tabular}{lllll}
\hline \multicolumn{1}{c}{ Família } & \multicolumn{3}{c}{ Período sazonal } \\
\cline { 3 - 5 } & Cheia & Vazante & Estiagem & Enchente \\
\hline Baetidae & $53 \pm 3.2$ & $0 \pm 3.2$ & $26 \pm 0.47$ & $9 \pm 4.7$ \\
Caenidae & $0 \pm 0$ & $0 \pm 0.9$ & $12 \pm 1.8$ & $2 \pm 1.8$ \\
Ceratopogonidae & $71 \pm 4.2$ & $77 \pm 1.88$ & $106 \pm 0.47$ & $59 \pm 2.3$ \\
Chironomidae & $301 \pm 0.94$ & $430 \pm 0.94$ & $860 \pm 1.88$ & $190 \pm 5.6$ \\
Culicidae & $6 \pm 0$ & $9 \pm 1$ & $40 \pm 5.1$ & $30 \pm 1.9$ \\
Helicopsychidae & $0 \pm 0$ & $10 \pm 3.77$ & $5 \pm 4.24$ & $3 \pm 0.47$ \\
Hidracarinidade & $74 \pm 4.5$ & $10 \pm 1$ & $12 \pm 0.5$ & $14 \pm 1.75$ \\
Hydroptilidae & $12 \pm 1.4$ & $84 \pm 0.47$ & $259 \pm 1.41$ & $14 \pm 0$ \\
Hydropsychidae & $13 \pm 5.1$ & $9 \pm 0.94$ & $36 \pm 55.1$ & $0 \pm 0$ \\
Leptophebiidae & $0 \pm 1.8$ & $6 \pm 0$ & $23 \pm 4.71$ & $3 \pm 1.41$ \\
Leptoceridae & $16 \pm 0.9$ & $0 \pm 0.94$ & $28 \pm 4.7$ & $29 \pm 1.41$ \\
Naididae & $100 \pm 1.03$ & $106 \pm 2.35$ & $57 \pm 2.8$ & $62 \pm 2.3$ \\
Psychodidae & $106 \pm 1.41$ & $55 \pm 0$ & $20 \pm 0$ & $1 \pm 0$ \\
Syrphidae & $119 \pm 0.94$ & $10 \pm 0$ & $1 \pm 0.47$ & $2 \pm 0$ \\
\hline
\end{tabular}

Assim como no estudo realizado por Labat (2017), díptera representou a maior riqueza, sendo ainda similar a abundância de Oligochaetos aqui representado pela família Naididae, presente em todas as baías e períodos do ciclo hidrológico

No presente estudo a família Chironomidae foi a mais abundante em todas as baías (Figura 3).Este fato está de acordo com a afirmação de Queiroz et al. (2014) indicando que esta família possui ampla distribuição geográfica e presença em todos os tipos de ambiente, aquático e semi-aquático.

Uieda et al. (2016) comparando dois mesohabitats encontrou em todos os ambientes estudados predominância da família Chironomidae, e presença de outros táxons, como Ephemeroptera, Trichoptera, assim como o presente trabalho. Barbosa et al. (2016) comparando dois métodos de coleta no rio Apodi- Mossoró obteve de forma abundante Moluscos, e na classe insecta, Libellulidae foi a mais representativa, já Chagas et al. (2017) Chironomidae e Hydropschidae, representaram as famílias mais abundantes em todos os pontos de coleta.

A enchente apresentou a menor abundância em relação aos outros períodos do ciclo hidrológico (Figura 2), com apenas 489 espécimes coletados; o que pode estar relacionado a desestabilização do sistema pelo aumento de vazão da água, o que promove um carreamento desses organismos, tendo alterações na profundidade e velocidade da água disponibilidade de micro habitats, e consequentemente na quantidade de macroinvertebrados (BISPO et al., 2001). 
No período da cheiaa média de OD, foi de $1,12( \pm 0.58) \mathrm{mg} / \mathrm{l}$. Neste mesmo período observou-se a terceira maior abundância de macroinvertebrados, Os níveis baixos de oxigênio dissolvido interferem na abundância (SANTOS et al., 2014, DAVANSO; HENRY, 2006), o que faz com que procurem oxigênio e abrigo junto às macrófitas (FULAN et al., 2014) o que explica a abundância encontrada.

A abundancia de Chironomidae e Naididae pode ser atribuída aos nichos apropriados para o estabelecimento e sobrevivência destas espécies nas raízes e folhas da macrófita (SANCHES; GORNI, 2014; SAULINO; TRIVINHO- STRIXINO, 2014), e a plasticidade ambiental que possuem (OLIVEIRA-JUNIOR et al., 2014).

Durante a vazante foi identificado a segunda abundância em relação ao período estudado, diferentemente de Silva et al. (2011) quando as menores quantidades de macroinvertebrados foram identificados durante a vazante. Com o escoamento e velocidade da água interferindo na abundância de organismos (SILVA et al., 2011).

A estiagem apresentou a maior abundância, com predominância das ordens Diptera, Trichoptera e Ephemeroptera, que diferentemente de Oliveira- Junior et al. (2014) relatou que a abundância de invertebrados foi maior durante a enchente e a cheia. Segundo os autores a dinâmica do pulso de inundação no pantanal afeta a densidade dos organismos e os períodos de águas altas aumentam a disponibilidade de alimento, o que pode determinar maiores abundâncias.

Segundo Fulan et al. (2014) a incidência de luz interfere na quantidade de OD, durante a estiagem, fazendo com que os raios solares atinjam camadas mais profundas da água, proporcionando alterações positivas na abundância de macroinvertebrados, assim como o pH próximo a neutralidade (SILVA; HENRY, 2013).

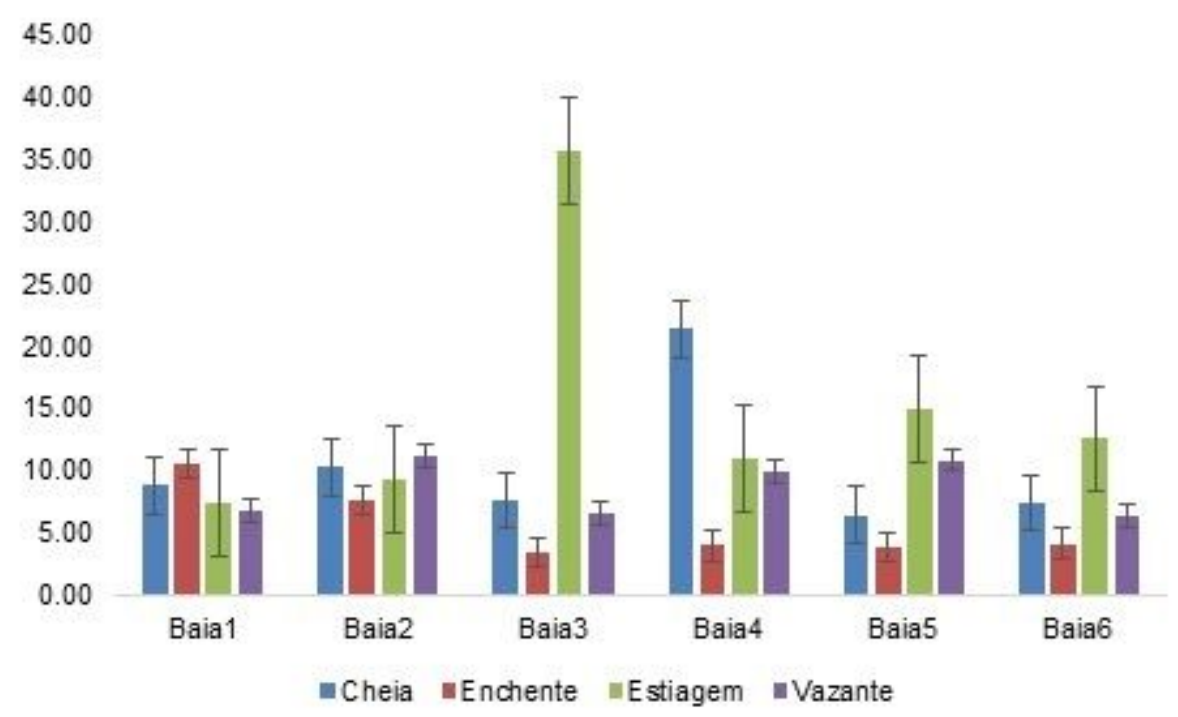

Figura 2. Valores médios de macroinvertebrados coletados no rio Paraguai. 2014. Cáceres, Mato Grosso. Fonte: Autores (2015).

As características Limnológicas variaram durante as fases do ciclo hidrológico; Oxigênio dissolvido variou de $\pm 1.12 \mathrm{mg} / \mathrm{l}$ na cheia à $\pm 5.74 \mathrm{mg} / \mathrm{l}$ na estiagem. Cesar e Henry (2017) no rio Guareí registrou alto OD em outubro inicio da vazante e baixa concentração de OD em abril, inicio da vazante. 
$\mathrm{O} \mathrm{pH}$ não apresentou grandes variações permanecendo ligeiramente ácido, próximo a neutralidade durante os quatro períodos. Gomes et al. (2017) encontrou $\mathrm{pH}$ levemente ácido e temperatura elevadas em todas as coleta. A temperatura da água apresentou uma variação de $\pm 5^{\circ} \mathrm{C}$ durante 0 ciclo hidrológico sendo que 0 período com maior temperatura foi registrado durante a enchente, e o com menor temperatura durante a vazante.

TABELA 3. Dados limnológicos das seis baías durante o ano de 2014. Cáceres-MT.

\begin{tabular}{lcccl}
\hline $\begin{array}{l}\text { Período } \\
\text { Sazonal }\end{array}$ & pH & OD $(\mathbf{m g})$ & Temp. água $\left({ }^{\circ} \mathbf{C}\right)$ & Transp. $\mathbf{C m}$ \\
\hline Cheia & $6.30 \pm 0.40$ & $1.12 \pm 0.58$ & $29.16 \pm 1.32$ & $78.90 \pm 37.30$ \\
Vazante & $6.63 \pm 0.28$ & $3.16 \pm 1.58$ & $25.94 \pm 1.99$ & $153.22 \pm 65.78$ \\
Estiagem & $6.95 \pm 0.37$ & $5.74 \pm 1.47$ & $27.18 \pm 2.82$ & $83.05 \pm 32.13$ \\
Enchente & $6.76 \pm 0.26$ & $3.43 \pm 2.24$ & $30.01 \pm 4.04$ & $65.38 \pm 29.03$
\end{tabular}

$\mathrm{pH}$ - Potencial hidrogeniônico; OD- Oxigênio Dissolvido; Temp. água $\left(\mathrm{C}^{\circ}\right)$ - Temperatura da água; Transp. Cm- Transparência da coluna d'água

A variação na abundância entre as fases do ciclo hidrológico e as baías pode ser uma influência dos parâmetros ambientais, a qual sofre com as mudanças dinâmicas de OD (SANTOS et al., 2014; MARÇAL et al., 2015), pH (SILVA; HENRY, 2013), velocidade da água (BISPO et al., 2001), turbulência e profundidade da água da região litoral (BEGHELLI et al., 2014).

As baías um, quatro e seis obtiveram os maiores índices de diversidade em Shannon-Wiener, e homogeneidade. Quando mensurado por fase do período hidrológico, a cheia e a vazante obtiveram os maiores índices de diversidade, tendo como variante a enchente, a qual apresentou o segundo maior índice de homogeneidade. Um ambiente menos perturbado é refletido pela diversidade, em que menos perturbação reflete em uma maior diversidade (COPATTI et al., 2014; CHAGAS et al., 2017), como pode ser observado na tabela 4.

TABELA 4 - Índices de diversidade $\left(\mathrm{H}^{\prime}\right)$ e homogeneidade $(\mathrm{IH})$ obtidos nas seis baías coletadas e nas quatro fases do ciclo hidrológico durante o ano de 2014. Cáceres-MT. Pantanal Norte

\begin{tabular}{lllllllllll}
\hline Índice B 1 & B 2 & B 3 & B 4 & B 5 & B 6 & Cheia Vaz & Est & Enc
\end{tabular}

\begin{tabular}{lllllllllll}
\hline $\mathrm{H}^{\prime}$ & 1.02 & 0.92 & 0.83 & 1.01 & 0.90 & 0.96 & 1.02 & 1.00 & 0.78 & 0.96 \\
& & & & & & & & & & \\
& 0.60 & 0.55 & 0.49 & 0.60 & 0.53 & 0.56 & 0.71 & 0.64 & 0.51 & 0.66
\end{tabular}

$\mathrm{IH}$

B1- Baía1; B2- Baía 2; B3- Baía 3; B4-Baía 4; B5- Baía5; B6- Baía 6; Vaz- Vazante. EstEstiagem; Enc- Enchente.

Barbola et al. (2011) utilizaram em associação os índice de diversidade e o índice biótico BMWP para obtenção da riqueza e o grau de conservação da área estudada, onde encontraram baixos índices de diversidade de famílias para macroinvertebrados em um reservatório na bacia do rio Pitangui no Paraná nas 
áreas consideradas pelo BMWP como boa, assim como neste estudo. $\mathrm{O}$ índice BMWP para os dados observados neste trabalho é apresentado na tabela 5.

TABELA 5 - Valores do índice BMWP obtidos através de coletas em seis baías. Pantanal Norte. 2014. Cáceres-MT.

\begin{tabular}{|c|c|c|c|c|c|c|c|}
\hline Táxon & BMWP & BAÍA 1 & BAÍA 2 & BAÍA 3 & BAÍA 4 & BAÍA 5 & BAÍA 6 \\
\hline \multicolumn{8}{|l|}{ Achari } \\
\hline Hidracarinidae & 4 & 6 & 10 & 11 & 14 & 16 & 7 \\
\hline \multicolumn{8}{|l|}{ Bivalva } \\
\hline Hyriidae & - & 3 & 3 & 1 & 1 & 2 & 0 \\
\hline \multicolumn{8}{|l|}{ Coelencerata } \\
\hline Hidridae & - & 59 & 10 & 1 & 58 & 4 & 1 \\
\hline \multicolumn{8}{|l|}{ Coleóptera } \\
\hline Dixidae & 4 & 0 & 0 & 1 & 0 & 0 & 0 \\
\hline Dytiscidae & 3 & 11 & 7 & 15 & 7 & 14 & 4 \\
\hline Elmidae & 5 & 4 & 2 & 0 & 0 & 0 & 1 \\
\hline Gyrinidae & 3 & 1 & 1 & 0 & 2 & 0 & 0 \\
\hline Halipidae & - & 2 & 0 & 0 & 2 & 0 & 0 \\
\hline Hydrophilidae & - & 13 & 2 & 0 & 10 & 3 & 1 \\
\hline Noteridae & - & 1 & 0 & 1 & 0 & 0 & 0 \\
\hline \multicolumn{8}{|l|}{ Diptera } \\
\hline Ceratopogonidae & 4 & 46 & 48 & 85 & 59 & 25 & 50 \\
\hline Chaboridae & - & 0 & 0 & 1 & 1 & 0 & 0 \\
\hline Chironomidae & 2 & 192 & 308 & 389 & 402 & 294 & 181 \\
\hline Culicidae & 2 & 24 & 11 & 0 & 10 & 6 & 14 \\
\hline Psychodidae & 4 & 24 & 27 & 31 & 44 & 39 & 11 \\
\hline Ptychopteridae & - & 0 & 13 & 0 & 0 & 13 & 0 \\
\hline Pyralidae & 7 & 0 & 0 & 3 & 0 & 0 & 3 \\
\hline Shyrphidae & 1 & 18 & 10 & 7 & 34 & 0 & 21 \\
\hline Tharidae & - & 0 & 0 & 1 & 0 & 0 & 0 \\
\hline Tipulidae & 5 & 1 & 0 & 0 & 0 & 0 & 0 \\
\hline \multicolumn{8}{|l|}{ Ephemeroptera } \\
\hline Baetidae & 5 & 11 & 7 & 16 & 44 & 11 & 13 \\
\hline Caenidae & 5 & 2 & 4 & 2 & 0 & 2 & 0 \\
\hline Leptophlebiidae & 10 & 8 & 9 & 4 & 26 & 2 & 10 \\
\hline Oligoneuriidae & - & 0 & 0 & 3 & 0 & 0 & 1 \\
\hline \multicolumn{8}{|l|}{ Gastropoda } \\
\hline Ampulariidae & - & 0 & 0 & 0 & 0 & 2 & 0 \\
\hline Ancylidae & 7 & 4 & 5 & 0 & 0 & 2 & 2 \\
\hline Planorbidae & 3 & 2 & 21 & 5 & 2 & 6 & 1 \\
\hline \multicolumn{8}{|l|}{ Hirudinea } \\
\hline Glossiphonidae & 3 & 0 & 8 & 6 & 0 & 0 & 1 \\
\hline Ozobranchidae & - & 14 & 9 & 18 & 34 & 10 & 4 \\
\hline \multicolumn{8}{|l|}{ Hemiptera } \\
\hline Belostomatidae & - & 2 & 1 & 0 & 0 & 1 & 0 \\
\hline Corixidae & 3 & 0 & 0 & 0 & 0 & 0 & 0 \\
\hline Pleidae & 3 & 0 & 0 & 1 & 0 & 2 & 0 \\
\hline Notononectidae & 3 & 0 & 1 & 0 & 0 & 0 & 0 \\
\hline \multicolumn{8}{|l|}{ Odonata } \\
\hline Dicteriadidae & - & 6 & 6 & 0 & 12 & 1 & 5 \\
\hline Gomphidae & 8 & 0 & 0 & 0 & 1 & 0 & 0 \\
\hline Libellulidae & 8 & 7 & 14 & 0 & 4 & 11 & 6 \\
\hline \multicolumn{8}{|l|}{ Oligochaeta } \\
\hline Naididae & 1 & 42 & 38 & 39 & 71 & 59 & 76 \\
\hline \multicolumn{8}{|l|}{ Trichoptera } \\
\hline Helicopsychidae & 10 & 4 & 6 & 0 & 3 & 2 & 3 \\
\hline Hidrobiosidae & 7 & 0 & 1 & 1 & 0 & 0 & 0 \\
\hline
\end{tabular}

ENCICLOPÉDIA BIOSFERA, Centro Científico Conhecer - Goiânia, v.15 n.28; p. 1050 


\begin{tabular}{llllllll} 
Hydropsychidae & 5 & 38 & 8 & 130 & 91 & 28 & 70 \\
Hydroptilidae & 6 & 17 & 26 & 2 & 20 & 24 & 13 \\
Leptoceridae & 10 & 5 & 8 & 10 & 6 & 2 & 3 \\
Nota BMWPX & - & $\mathbf{5 6 9}$ & $\mathbf{6 9 1}$ & $\mathbf{8 4 6}$ & $\mathbf{9 2 9}$ & $\mathbf{6 3 7}$ & $\mathbf{5 9 0}$ \\
\hline
\end{tabular}

Todas as baías possuem segundo o índice biótico BMWP um alto grau de conservação, não apresentando sinais de poluição, encaixando-se na classe I com a cor de indicação azul. Os menores índices obtidos foram 569 pela baía 1 e 590 pela baía 6, enquanto os maiores índices, 846 e 929 foram obtidos pelas baías três e quatro respectivamente (Tabela 3 ).

O BMWP apontou que entre os períodos sazonais a estiagem com 1753 demonstrou melhor qualidade da água, seguido da vazante 1279 e a Cheia 1155, a enchente obteve o menor índice 619 , indicando boa condição do sistema. A variação das notas do BMWP durante os períodos sazonais é atribuída as alterações decorrentes do ciclo hidrológico no Pantanal (OLIVEIRA-JUNIOR et al., 2014), que afeta e altera a comunidade de macroinvertebrados.

O número de famílias coletadas e a presença de famílias sensíveis a alterações ambientais indicam elevado grau de conservação (SILVA et al., 2011), o que corrobora com os valores encontrados no BMWP e apontam para a qualidade da água nas seis baías coletadas e durante o ciclo hidrológico estudado.

As menores notas obtidas no BMWP contrastaram com os índices de diversidade e homogeneidade obtidos. Santos-Silva et al. (2016) obtiveram os índices de diversidade mais alto nos pontos com a nota BMWP mais altas, diferentemente dos valores encontrados no presente trabalho onde apenas a baía 1 dentre as baías com maior índice de diversidade obteve também nota alta no BMWP, demonstrando que a região pantaneira tem dinâmica própria em relação a sua macrofauna fitófila. Assim, inferimos que os macroinvertebrados associados as macrófitas marginais as baías pantaneiras também podem servir como base para a utilização dos índices de identificação ambiental e consequentemente destacando sua qualidade.

\section{CONCLUSÃO}

De acordo com os índices obtidos as baías apresentam uma boa qualidade da água. A diversidade de famílias de macroinvertebrados encontrados demonstra a riqueza da fauna fitófila pantaneira. Como mostrou nosso estudo as baías marginais apresentam boa qualidade da água, com o BMWP alto em todas as baías, e abundância em diferentes fases do ciclo hidrológico.. Novos estudos sobre a macrofauna do Pantanal ainda são necessários, bem como o monitoramento da qualidade das águas. Ainda, devido à escassez de conhecimento na área, dados pioneiros como este auxiliam no desenvolvimento da ciência em busca de alternativas de análise ambiental e avaliação estratégica. Demonstramos aqui dados que identificam o ambiente pantaneiro, e alertamos para que as modificações antrópicas que venham a ocorrer podem também ocasionar uma mudança na estrutura da comunidade, consequentemente alterando a qualidade ambiental.

\section{AGRADECIMENTOS}

Agradecemos ao Projeto Bichos do Pantanal, através do Programa Petrobras Socioambiental pelo apoio e financiamento. 


\section{REFERÊNCIAS}

ALBA-TERCEDOR, J.; SÁNCHEZ- ORTEGA, A.; Un método rápido y simple para 295 evaluar la calidad biológica de las aguas corrientes basado em el de Hellawell (1978). 296 Limnética, v. 4, n. 1. p. 51-56. 1988. Disponível em: http://www.limnetica.com/documentos/limnetica/limnetica-4-1-p-51.pdf

BARBA-ÁLVAREZ, R.; LANZA-ESPINO, G.; CONTRERAS-RAMOS, A.; GONZÁLEZ-MORA, I,; Insectos acuáticos indicadores de calidad del agua en México: casos de estudio, ríos Copalita, Zimatán y Coyula, Oaxaca. Revista Mexicana de Biodiversidade v. 84 p. 381-383. 2013. Disponível em: DOI: 10.7550/rmb.31037> DOI: 10.7550/rmb.31037

BARBOLA, I. F.; MORAES, M. F. P. G.; TATHIANE M. ANAZAWA, T. M.; NASCIMENTO, E. A.; SEPKA, E. R.; POLEGATTO, C. M.; MILLÉO, J.; SCHÜHLI, G. S.; Avaliação da comunidade de macroinvertebrados aquáticos como ferramenta para o monitoramento de um reservatório na bacia do rio Pitangui, Paraná, Brasil. Iheringia, Série Zoologia, Porto Alegre. v. 1-2 p.15-23. 2011. Disponível em: http://dx.doi.org/10.1590/S0073-47212011000100002>ＤOl.org/10.1590/S007347212011000100002

BARBOSA, A. H. S.; SILVA, C. S. P.; ARAÚJO, S. E.; LIMA, T. B. B.; DANTAS, I. M.; Macroinvertebrados bentônicos como bioindicadores da qualidade da água em um trecho do rio Apodi-Mossoró. HOLOS, Ano 32, v. 7. 2016. Disponível em: DOI: 10.15628/holos.2016.4183 DOI: 10.15628/holos.2016.4183.

BAZZANTI, M.; COCCIA, C.; DOWGIALLO, M.G.; Microdistribution of macroinvertebrates in a temporary pond of Central Italy: taxonomic and functional analyses. Limnologica Ecology and Management of Inland Waters, v. 40, p. 291299. 2010. Disponível em: https://doi.org/10.1016/j.limno.2009.10.006> doi.org/10.1016/j.limno.2009.10.006

BISPO, P. C.; OLIVEIRA, L. G.; CRISCI, V. L.; SILVA, M. M.; A pluvisiodade como fator de alteração da entomofauna Bêntonica (Ephemeroptera, Plecoptera e Trichoptera) em córregos do Planalto Central do Brasil. Acta Limnologica

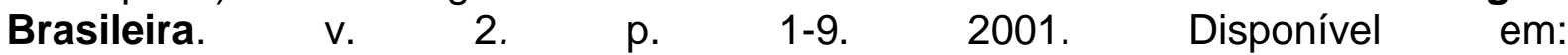
http://www.ablimno.org.br/acta/pdf/acta_limnologica_contents1302E_files/Artigo\%20 1_13(2).pdf

CALOR, A.R. Trichoptera. In: Guia on-line de Identificação de larvas de Insetos Aquáticos do Estado de São Paulo. 2007. Disponível em: http://sites.ffclrp.usp.br/aguadoce/index trico Acesso em 23 de fevereiro de 2014.

CESAR, D. A. S.; HENRY, R.; Is similar the distribution of Chironomidae (Diptera) and Oligochaeta (Annelida, Clitellata) in a river and a lateral fluvial area? Acta Limnologica Brasiliensia, v. 29. 2017. Disponível em: http://dx.doi.org/10.1590/S2179-975X1217 DOl.org/10.1590/S2179-975X1217.

CHAGAS, F. B.; RUTKOSKI, C. F.; BIENICK, G. B.; VARGAS, G. D. P. L.; HARTMANN, P. A.; HARTMANN, M. T.; Utilização da estrutura de comunidades de 
macroinvertebrados bentônicos como indicador de qualidade da água em rios no sul do Brasil. Revista Ambiente e Água. v. 12 n. 3. 2017. Disponível em: DOI:10.4136/ambi-agua.2015 DOI:10.4136/ambi-agua.2015.

COPATTI, C. E.; MOREIRA, T. B.; MENZEL, C. A.; Avaliação da qualidade ambiental de uma microbacia no sul do Brasil através de diferentes abordagens. Ambiência. v. 10 n. 2 p. 511-526. 2014. Disponível em: DOI:10.5935/ambiencia.2014.02.06 DOI:10.5935/ambiencia.2014.02.06.

CORTEZZI, S. S.; BISPO, P. da C.; PACIENCIA, G. de P.; LEITE, R.C.; Influência da ação antrópica sobre a fauna de macroinvertebrados aquáticos em riachos de uma região de cerrado do sudoeste do Estado de São Paulo. Iheringia, Série Zoologia. Porto Alegre. v. 1. p. 36-43. 2009. Disponível em: http://dx.doi.org/10.1590/S007347212009000100005 http://dx.doi.org/10.1590/S0073-47212009000100005.

DAVANSO, R. C. S.; HENRY, R.; A biodiversidade bentônica em lagoa marginal ao rio Paranapanema na zona de sua desembocadura, na represa de Jurumirim. Acta Scientarum Biology Science v. 28, n. 4. p. 347-357. 2006. Disponível em: DOI: 10.4025/actascibiolsci.v28i4.166> DOI: 10.4025/actascibiolsci.v28i4.166.

FROTA, A.V.B.; IKEDA-CASTRILLON, S. K.; KANTEK, D. L. Z.; DA SILVA, C. J.; Macrohabitats da Estação Ecológica de Taiamã, no contexto da Área Úmida Pantanal mato- grossense, Brasil. Boletim Paraense Emílio Goeldi Ciências Naturais. Belém, v. 12, n. 2, p. 239-254. 2017. Disponível em: http://www.museugoeldi.br/editora/bn/artigos/cnv12n2_2017/macrohabitats(frota).pdf

FULAN, J. Â.; DE MENEZES, J. A.; DA SILVA, V. V.; Migração vertical de macroinvertebrados entre o sedimento e a macrófita Salvinia auriculata Aublet. Biológico, São Paulo, v. 76, n. 1, p. 69-76. 2014. Disponível em: http://biologico.agricultura.sp.gov.br/uploads/docs/bio/v76_1/fulan.pdf

GOMES, D. F.; SANCHES, N. A. O.; SAHM, L. H.; GORNI, G. R.; Aquatic oligochaeta (Annelida: Clitellata) in extractive reserve Lake Cuniã, Western Brazilian Amazon. Biota Neotropica v. 17 n. 1. 2017. Disponível em: Biota Neotropica 17(1): e20160232, 2017

LABAT, F.; A new method to estimate aquatic invertebrate diversity in French shallow lakes and ponds. Ecological Indicators v. 81. p. 401-408. 2017. Disponível em: https://doi.org/10.1016/j.ecolind.2017.05.073> doi.org/10.1016/j.ecolind.2017.05.073

MARÇAL, S. F.; CALIL, C. T.; SANTOS, R. C. L.; Trichoptera (Insecta) in water hyacinth roots: evaluation of the influence of exotic mussel and environmental factors. Acta Limnologica Brasiliensia, v. 27 n. 4. p. 341-355. 2015. Disponível em: http://dx.doi.org/10.1590/S2179-975X0715 doi.org/10.1590/S2179-975X0715

MARTINS, R. T.; SILVEIRA, L. S.; ALVES, R. G. Colonization by oligochaetes (Annelida: Citellata) in decomposing leaves of Eichhornia azurea (SW.) Kunth (Pontederiaceae) in a tropical lentic system. Annales de Limnologie - International 
Journal of Lymnologi, Santa Fé, v. 47, p. 339-346. 2011. Disponível em: DOI: 10.1051/limn/2011053 DOI: 10.1051/limn/2011053

MUGNAI, R.; NESSIMIAN, J. L.; BAPTISTA, D.F.; Manual de Identificação de Macroinvertebrados Aquáticos do Estado do Rio de Janeiro; p.174. 1ํำ Edição. 2010.

OLIVEIRA-JUNIOR, E. S.; MUNIZ, C. C.; BUTAKKA, C. M. de M.; Invertebrados bentônicos de uma baía do Pantanal mato-grossense, MT: comunidade determinada pela dinâmica das águas. Caderno de Pesquisa, série Biologia, v. 26, n. 2. p. 16-24. 2014. Disponível em: DOI: http://dx.doi.org/10.17058/cp.v26i2.4739 DOI: http://dx.doi.org/10.17058/cp.v26i2.4739

PAGIORO, T.A.; THOMAZ, S.M.; Influence of the decomposition of Eichhornia azurea on selected abiotic limnological variables of different environments of the floodplain of the High Paraná River. Acta Limnologica Brasiliensia, v. 2. p. 157171. 1999.

Disponível em: http://www.ablimno.org.br/acta/pdf/acta_limnologica_contents1102E_files/Artigo\%20 13_11(2).pdf

PAIVA, S. L. P.; NEVES, S. M. A. S.; MUNIZ, C. C.; NEVES, R. J.; KREITLOW, J. P.; Análise de ocorrência de queimadas em unidades geomorfológicas na bacia Amazônica, contida na região sudoeste mato-grossense. Revista Geonorte, Edição Especial 4, v. 10, n. 1, p. 95-100, 2014. Disponível em <http://www.periodicos.ufam.edu.br/revista-geonorte/article/view/1660>

PES, A. M. O.; HAMADA, N.; NESSIMIAN, J.L.; Chaves de identificação de larvas para famílias e gêneros de Trichoptera (Insecta) da Amazônia Central, Brasil. Revista Brasileira de Entomologia. v. 2. p. 181-204. 2005. Disponível em: http://dx.doi.org/10.1590/S0085-56262005000200002> doi.org/10.1590/S008556262005000200002.

PERES, P. N.; MIOTO, C. L.; MARCATO-JUNIOR, J.; PARANHOS-FILHO, A. C.; Variação da Cobertura do Solo no Pantanal de 2000 a 2015 por Sensoriamento Remoto com Software e Dados Gratuitos. Anuário do Instituto de Geociências UFRJ- v. 39. n. 2. p. 116-123. 2016. Disponível em: DOI: http://scihub.tw/10.11137/2016_2_116_123> DOI: http://scihub.tw/10.11137/2016_2_116_123

PÉREZ, G. R.; Guía para el estúdio de los macroinvertebrados acuáticos del departamento de Antioquia. Bogotá. Fondo Colombiano de investigaciones Científicas y Proyectos Especiales "Francisco José de Caldas", Universidad de Antioquia. p. 217. 1988.

PIRES, E.; ANDRADE, L. N. P. S.; SOARES, J. C. O.; LEANDRO, G. R. S.; Impactos ambientais na margem direita do rio Jauru no bairro Beira Rio, Porto Esperidião - Mato Grosso. Revista Brasileira de Geografia Física. v. 07 n. 03. p. 
540-557.

2014.

Disponível

em:

https://periodicos.ufpe.br/revistas/rbgfe/article/view/233123/27038

QUEIROZ, J. F.; TRIVINHO-STRIXINO, S.; NASCIMENTO, V.M.C.; Organismos bentônicos bioindicadores da qualidade das águas da bacia do médio São Francisco. 2000. Comunicado Técnico. Embrapa Informática Agropecuária. №. 3, novembro. Disponível em www.cnpma.embrapa.br/dowload/organ betonicos.pdf Acesso em 23 de fevereiro de 2014.

RODRIGUES-JUNIOR, U. J.; BAMPI, A. C., CRUZAN, J. S.; A atenuação dos impactos e conscientização dos agentes envolvidos no processo de uso e ocupação das matas ciliares no rio Paraguai: Entre a foz do rio Sepotuba e a cidade de Cáceres região do Pantanal mato-grossense através da gestão ambiental. Revista de Estudos Sociais. v. 15. n. 30. p. 3. 2013. Disponível em: http://www.periodicoscientificos.ufmt.br/ojs/index.php/res/article/viewFile/2044/pdf

ROLDÁN-PEREZ, G.; Los macroinvertebrados como bioindicadores de la calidad del agua: cuatro décadas de desarrollo en Colombia y Latinoamerica. Revista de la Academia Colombiana de Ciencias Exactas, Físicas y Naturales. v. 40. p. 254274. 2016. Disponível em: doi: http://dx.doi.org/10.18257/raccefyn.335 DOI: doi: http://dx.doi.org/10.18257/raccefyn.335

ROMERO, K. C.; DEL RIO, J. P.; VILLARREAL, K. C.; ANILLO, J. C. C.; FRANCO, O. L.; VALENCIA, J. W. A.; Lentic water quality characterization using macroinvertebrates asbioindicators: An adapted BMWP index. Ecological Indicators v. 72 p. 53-66. 2017. Disponível em: http://dx.doi.org/10.1016/j.ecolind.2016.07.023 DOI: http://dx.doi.org/10.1016/j.ecolind.2016.07.023

SANCHES, H. A. O.; GORNI, G. R.; Preferência de habitat de Oligochaeta (Annelida, Clitellata) em macrófitas aquáticas na Represa Ribeirão das Anhumas (Américo Brasiliense-SP). Revista Uniara, v. 17 p. 165-173. 2014. Disponível em: DOI: https://doi.org/10.25061/2527-2675/ReBraM/2014.v17i1.16> https://doi.org/10.25061/2527-2675/ReBraM/2014.v17i1.16.

SANTOS, L. R.; FERREIRA, L.; FULAN, J. A. Levantamento da riqueza de macroinvertebrados que habitam as macrófitas e o sedimento no Igarapé por alunos de uma escola pública de Humaitá, AM. Revista Ciência em Extensão. v. 10 n. 3, p. $85-91$ 2014.

Disponível

em:

http://ojs.unesp.br/index.php/revista_proex/article/view/872/1036http://ojs.unesp.br/in dex.php/revista_proex/article/view/872/1036.

SANTOS-SILVA, K. W.; MELO, M. A. D.; EVERTON, N. S.; Aplicação dos índices biológicos Biological Monitoring Working Party e Average Score per Taxon para avaliar a qualidade de água do rio Ouricuri no Município de Capanema, Estado do Pará, Brasil. Revista Pan-Amazonica Saude v. 7 n. 3. p. 13-22. 2016. Disponível em: DOI: 10.5123/S2176-62232016000300002> DOI: 10.5123/S217662232016000300002. 
SAULINO, H. H. L.; TRIVINHO-STRIXINO, S.; Macroinvertebrados aquáticos associados às raízes de Eichhornia azuera (Swarts) Kunth (Pontederiaceae) em uma lagoa marginal no Pantanal, MS. Revista Biotemas, v. 3. p. 65-72. 2014. Disponível em: DOI: https://doi.org/10.5007/2175-7925.2014v27n3p65

DOI: https://doi.org/10.5007/2175-7925.2014v27n3p65.

SILVA, F. H.; FAVERO, S.; SABINO, J.; GARNÉS, S. J. A.; Índices bióticos para avaliação da qualidade ambiental em trechos do rio Correntoso, Pantanal do Negro, Estado do Mato Grosso do Sul, Brasil. Acta Scientiarum Biological Sciences. Maringá, v. 33, n. 3, p. 289-299. 2011. Disponível em: doi: 10.4025/actascibiolsci.v33i3.1478> DOI: 10.4025/actascibiolsci.v33i3.1478.

SILVA, C.V.; HENRY, R.; Aquatic macroinvertebrates associated with Eichhornia azurea (Swartz) Kunth and relationships with abiotic factors in marginal lentic ecosystems (São Paulo, Brazil). Brazilian Journal of Biology. v. 73, n. 1, p. 149162. 2013. Disponível em: http://dx.doi.org/10.1590/S1519-69842013000100016 http://dx.doi.org/10.1590/S1519-69842013000100016.

SOUZA-FILHO, E. E.; As barragens na bacia do rio Paraguai e a possível influência sobre a descarga fluvial e o transporte de sedimentos. Boletim geográfico. Maringá. v. 31. n. 1. p. 117-133. 2013. Disponível em: doi: 10.4025/bolgeogr.v31i1.13638> DOI: 10.4025/bolgeogr.v31i1.13638.

THOMAZ, S. M.; PAGIORO, T. A.; PADIAL, A. A.; 2003. Macrófitas aquáticas. Disponível em: www.peld.uem.br/Relat2003/pdf/Macrofitas_aquaticas.pdf . Acesso em 12 de janeiro de 2015.

UIEDA, V. V.; ALVES, M. I. B.; SILVA, E. I.; Invertebrados bentônicos: relação entre estrutura da fauna e características do mesohabitat. Revista Ambiente e Água. v. 11 n. 3. 2016. Disponível em: http://dx.doi.org/10.4136/ambi-agua.1876 doi.org/10.4136/ambi-agua.1876.

YOSHIDA, C. E.; UIEDA, V. S.; Índices bióticos mono e multimétricos de avaliação da qualidade da água em riachos de Mata Atlântica. Bioikos, Campinas, v. 27 n. 2. p. 79-88, 2013. Disponível em: https://www.researchgate.net/publication/269920535/download. 